\title{
MỐI TƯƠNG QUAN CỦA SỰ TÍCH TỤ Mỡ BỤNG TRÊN HİNH ẢNH SIÊU ÂM VớI CHỈ Số NHÂN TRẮC Ở BÊ̂NH NHÂN GAN NHIỄM MÕ̃
}

\author{
Nguyễn Thị Sinh*, Trịnh Xuân Đàn*, \\ Nguyễn Văn Quỳnh**, Nguyễn Thị Bình*, \\ Hoàng Thị Lệ Chi*, Đoàn Thị Nguyệt Linh*
}

\section{TÓM TẮT}

Mục tiêu: Xác định mối tương quan của sự tích tụ mỡ bụng trên hình ả̉nh siêu âm với nhân trắc học ở bệnh nhân gan nhiễm mỡ. Đối tượng và phương pháp: Nghiên cựu mô tả cắt ngang trên 200 bệnh nhân có gan nhiễm mõ̃ và có gan không nhiễm mõ bằng đo các chỉ tiêu: chiều cao, cân nặng, vòng bụng, vòng mông, chỉ số BMI, Tỷ lệ Vòng bụng/Vòng mổng, độ dày lớp mõ dưới da (ĐDLMDD), độ dày lớp mõ trước phúc mạc (ĐDLMTPM), độ dày lớp mõ nội tạng (ĐDLMNT). Kết quả và kết luânn: Độ dày lớp mõ̃ dưới da với Độ dày lớp mõ trước phúc mạc $(r=0,23$; $p<0,05)$ và Độ dày lớp mõ dưới da với độ dày lớp mṍ nội tạng $(r=0,18 ; p<0,05)$ tương quan ở mức thấp; ĐDLMNT với ĐDLMTPM $(r=0,42 ; p<0,05)$ tương quan ở mức trung bình. Tương quan thuận ở mức trung bình giữa ĐDLMNT với các chỉ tiêu cẩn nặng ( $r$ $=0,48)$, vòng bụng $(r=0,47)$ và chỉ số BMI $(r=$ 0,42 ) với $p<0,05$; tương quan thuận ở mức thấp giữa ĐDLMDD, ĐDLMTPM với chiều cao, cân nặng, vòng bụng và chỉ số BMI, với $p>0,05$.

Từ khóa: Siêu âm; Mõ bụng; Gan nhiễm mõ.

\section{SUMMARY}

CORRELATION OF ABDOMINAL FAT

ACCUMULATION ON ULTRASOUND IMAGES WITH ANTHROPOMETRIC INDEX IN LIVER STEATOSIS PATIENTS

Objectives: Determine the correlation of abdominal fat accumulation on ultrasound images with anthropometry in liver steatosis patients. Subjects and methods: The descriptive study cross-sectioned 200 patients with fatty liver and non-fatty liver by measuring indicators: height $(\mathrm{H})$, weight $(\mathrm{W})$, waist circumference $(W)$, buttocks (B) circumference, BMI, waist / buttocks ratio, subcutaneous fat thickness (S), peritoneal fat thickness $(P)$, visceral fat thickness $(\mathrm{V})$. Result and conclusion: Subcutaneous fat thickness with peritoneal fat thickness $(r=0.23 ; p<0.05)$ and Subcutaneous fat thickness with visceral fat thickness $(r=0.18 ; p<0.05)$ low correlation; peritoneal fat thickness with visceral fat thickness $(r=0.42 ; p$ $<0.05)$ medium correlation. Medium positive correlation between visceral fat thickness with weight $(r=0.48)$, waist circumference $(r=0.47)$ and BMI $(r$

\footnotetext{
*Trường $Đ H$ Y Dược Thái Nguyên

**Trường Cao đắng Y tế Thái Nguyên

Chịu trách nhiệm chính: Nguyễn Thị Sinh

Email: sinhnguyen.gp@gmail.com

Ngày nhận bài : 31/1/2021

Ngày phản biện khoa học : 25/2/2021

Ngày duyệt bài: 15/3/2021
}

$=0.42), p<0.05$; There is a low positive correlation between subcutaneous fat thickness (S), peritoneal fat thickness $(P)$ with height $(H)$, weight $(W)$, waist circumference $(\mathrm{W})$, buttocks $(B)$ circumference, BMI, p> 0.05 .

Keywords: Ultrasound; Abdominal fat; Liver steatosis

\section{I. ĐẠT VẤN ĐỀ}

Hiện nay, gan nhiễm mõ đang ngày càng phổ biến. Khoảng 10-20\% dân số Mỹ mắc gan nhiễm mõ, đa phân các trường hợp gan nhiễm mõ nằm trong độ tuổi từ 40-60. Chúng ta cân phát hiện sớm và điều trị thích hợp trước khi có những tổn thương gan thực sự. Đến nay, y học vẫn chuộng dùng siêu âm để chẩn đoán gan nhiễm mỡ do độ nhạy, độ đặc hiệu cao, an toàn và chi phí thấp.

Tuy nhiên, đối với bệnh nhân bị béo phì, mắc các bệnh gan mãn tính khác thì việc chẩn đoán cân phải được cân nhắc kỹ lưỡng. Thường thì các bác sĩ có xu hướng đưa ra chẩn đoán bệnh lý này tương đối dễ vì chỉ dựa vào hình ảnh gan sáng hơn bình thường nên khó tránh được xác suất nhầm lẫn. Nên nếu nghi ngờ vê tính chính xác của mô tả gan nhiễm mõ̃ trên siêu âm, bác sĩ có thể thực hiện kiểm tra bằng một phương pháp khác như đo vòng bụng, vòng mông để có kết quả độc lập và khách quan hơn.

Một nghiên cứu đã được thực hiện trên một nhóm gôm 50 phụ nữ béo phì với chỉ số khối cơ thể trung bình là $39 \pm 16$, từ 19 đến 60 tuổi, để xác định liệu siêu âm có thể được sử dụng như một phương tiện trực tiếp để đo lượng mõ trong bụng. Đánh giá mô mõ được thực hiện bằng chụp cắt lớp vi tính (CT Scannner) và siêu âm (sử dụng đâuu dò 3,5 - MHz và $5-\mathrm{MHz}$ ) ở mức L4. CT mõ vùng bụng (VAT) và siêu âm nội tạng độ dày từ cơ bụng đến động mạch chủ là những giá trị chủ yếu được xem xét. Siêu âm độ dày trong bụng tương quan tốt với VAT ( $r=0,669, p$ $<0,001)$, ủng hộ giả thuyết rằng siêu âm có thể hữu ích trong việc đánh giá trực tiếp mõ trong bụng. [1]

Để thêm bằng chứng cho việc xác định liệu siêu âm có thể được sử dụng như một phương tiên trực tiếp để đo lượng mỡ trong bụng; là một phương pháp hữu ích để ước tính mô mõ trong ổ bụng hay không và có liên quan đến các yếu tố 
như béo phì và hội chứng chuyển hóa hay không? Đề tài này nhằm mục tiêu: Xác định mối tương quan của sư tích tụ mõ bụng trên hinh ảnh siêu âm với nhân trắc học ở bệnh nhân gan nhiễm mõ

\section{II. ĐỐI TƯợNG VÀ PHƯƠNG PHÁP NGHIÊN CỨU}

2.1. Đối tượng nghiên cứu: Bệnh nhân từ 18 tuổi đến khám bệnh tại phòng siêu âm- bệnh viện trường đại học y khoa Thái Nguyên và được chẩn đoán gan nhiễm mõ̃ trên hình ảnh siêu âm.

- Tiêu chuẩn loại trừ: Bệnh lý gan khác, không đồng ý tham gia vào nghiên cứu.

- Địa điểm nghiên cứu: Bệnh viện Trường Đại học Y khoa Thái Nguyên

- Thời gian nghiên cứu: từ tháng 2/2020 đến tháng 12/2020.

2.2. Phương pháp nghiên cứu: Nghiên cứu mô tả cắt ngang

2.3. Kỹ thuật thu thập số liệu:

- Chỉ tiêu chung: Tuổi, giới

- Kỹ thuật đo đạc theo tiêu chuẩn trong nhân trắc học.

+ Cân năng: Đo bằng cân bàn Trung Quốc, tính đến $0,1 \mathrm{~kg}$

+ Chiêu cao: Đo bằng thước dây, tính đến 0,1cm.
+ Chỉ số khối cơ thể (BMI) được tính bằng:

BMI = cân nặng $(\mathrm{kg}) /[$ chiều cao (mét) $\mathrm{x}$ chiều cao (mét)].

+ Vòng bụng, vòng mông: Đo bằng thước dây, tính đến $0,1 \mathrm{~cm}$.

+ Tỷ lệ Vòng bụng /Vòng mông (VB/VM)

- Đọc kết quả siêu âm bởi các bác sĩ chuyên khoa

+ Chỉ tiêu siêu âm: Sử dụng máy siêu âm Phillip. Đầu dò mảng tuyến tính $(7,5 \mathrm{MHz}$ và 3,5 $\mathrm{MHz}$ ) được giữ vuông góc với da ở vùng bụng trên rốn. Độ dày mõ dưới da tối đa (ĐDLMDD) được đo ở mức $5 \mathrm{~cm}$ trên đường trắng giữa trên rốn; Đo lớp mõ trước phúc mạc (ĐDLMTPM) được lấy từ vùng mà nó được nhìn thấy rõ nhất ngay bên dưới đường trên rốn; Dùng đầu dò mảng lồi $(3,5 \mathrm{MHz})$ đã được sử dụng để đo độ dày lớp mõ nội tạng (ĐDLMNT) được lấy giữa mặt trong của cơ ngang bụng và thành trước của động mạch chủ. Tất cả các bệnh nhân được yêu cầu nín thở trong khi kiểm tra, đặc biệt cẩn thận để giữ cho đầu dò chỉ chạm vào da để ngăn chặn sự chèn ép của các lớp mõ [2].

- Phương pháp xử lý số liệu: Bằng phần mềm thống kê y học SPSS 22.00.

\section{KẾT QUẢ NGHIÊN CỨU}

3.1. Đặc điểm của đối tượng nghiên cứu

Bảng 3.1. Đăc điểm của các đối tượng nghiên cứu

\begin{tabular}{|c|c|c|c|c|c|c|}
\hline \multirow{2}{*}{$\begin{array}{l}\text { Đối tượng } \\
\text { Đặc điểm }\end{array}$} & \multicolumn{2}{|c|}{$\begin{array}{l}\text { Nhóm bệnh } \\
(n=100)\end{array}$} & \multirow[t]{2}{*}{ p } & \multicolumn{2}{|c|}{$\begin{array}{c}\text { Nhóm chứng } \\
(n=100)\end{array}$} & \multirow[t]{2}{*}{$\mathbf{p}$} \\
\hline & $\operatorname{Nam}(n=70)$ & $N \tilde{u}^{\prime}(n=30)$ & & $\operatorname{Nam}(n=70)$ & Nũ̃ $(n=30)$ & \\
\hline Tuổi & $43,16 \pm 13,27$ & $55,23 \pm 14,45$ & $<0,05$ & $45,07 \pm 7,71$ & $44,13 \pm 5,44$ & $<0,05$ \\
\hline Chiều cao & $1,67 \pm 0,07$ & $1,55 \pm 0,05$ & $<0,05$ & $1,66 \pm 0,05$ & $1,57 \pm 0,05$ & $<0,05$ \\
\hline Cân nặng & $70,36 \pm 11,43$ & $58,07 \pm 7,78$ & $<0,05$ & $62,65 \pm 6,76$ & $56,23 \pm 7,10$ & $<0,05$ \\
\hline Vòng bụng & $87,44 \pm 8,20$ & $80,7 \pm 8,56$ & $<0,05$ & $80,50 \pm 6,56$ & $76,00 \pm 6,12$ & $<0,05$ \\
\hline Vòng mông & $97,51 \pm 7,29$ & & & $93,27 \pm 5,26$ & $90,63 \pm$ & $<0,05$ \\
\hline & $9 \pm 2,98$ & $24,07 \pm 2,81$ & $<0,05$ & $22,75 \pm 2,19$ & $22,72 \pm 2,86$ & $<0,05$ \\
\hline Tỷ lệ VB/VM & $0,90 \pm 0,05$ & $0,87 \pm 0,08$ & $<0,05$ & $0,86 \pm 0,05$ & $0,84 \pm 0,01$ & $<0,05$ \\
\hline
\end{tabular}

Nhận xét: Bảng 3.1 đưa ra giá trị trung bình SD đối với kết quả nhân trắc học ở những đối tượng gan nhiễm mõ̃. Sự khác biệt đáng kể nhất giữa nhóm gan nhiễm mõ̃ và nhóm gan không nhiễm mõ̃ được thể hiện qua tuổi của nhóm gan nhiễm mõ ở nữ $(55,23 \pm 14,45)$; cân nặng, vòng bụng ở nhóm nam gan nhiễm mõ cao hơn nhóm nam gan không nhiễm mõ; chỉ số BMI ở nhóm bệnh cao hơn nhóm chứng nhưng vẫn trong giới hạn bình thường; vòng bụng của nữ nhóm bệnh lớn $(80,7 \pm 8,56)$ nhóm chứng, sự khác biệt có ý nghĩa thống kê với $p<0,05$ [3]

Bảng 3.2. Chi tiêu nhân trắc trên siêu âm của các đối tượng nghiên cứu

\begin{tabular}{|c|c|c|c|c|c|c|}
\hline \multirow{2}{*}{ Chỉ tiêu } & \multicolumn{2}{|c|}{ Nhóm bênh(n=) } & \multirow[b]{2}{*}{$\mathbf{P}$} & \multicolumn{2}{|c|}{ Nhóm chứng(n=) } & \\
\hline & $\operatorname{Nam}(n=)$ & Nữ $(n$ & & $\operatorname{Nam}(n=)$ & Nữ $(n=)$ & \\
\hline & & & $<0$, & & & \\
\hline & & & & & & \\
\hline ĐDLM NT (mm) & $31,53 \pm 12,03$ & $23,91 \pm 6,17$ & $<0,05$ & $15,57 \pm 5,14$ & $16,65 \pm 4,79$ & $<0,05$ \\
\hline
\end{tabular}

Nhận xét: Bảng 3.2 đưa ra kết quả các chỉ tiêu đo trên siêu âm cho thấy:

Ơ nam giới nhóm bệnh có các chỉ tiêu ĐDLMDD, ĐDLMTPM, ĐDLMNT là cao hơn so với nhóm 
chứng, có ý nghĩa thống kê $(p<0,05)$.

Ớ nhóm bệnh nữ các chỉ tiêu ĐDLMDD, ĐDLMTPM, ĐDLMNT cũng đều cao hơn nhóm chứng có ý nghĩa thống kê $((p<0,05)$.

3.2. Mối tương quan của sự tích tụ mõ̃ bụng với một số chỉ số nhân trắc

Bảng 3.3. Mối tương quan giữa các độ dày lớp mỡ với nhau ở bệnh nhân gan nhiễm mõ.

\begin{tabular}{|c|c|c|}
\hline & $\mathbf{r}$ & $\mathbf{p}$ \\
\hline & 0,23 & $<0,05$ \\
\hline ĐDLMDD với ĐDLMTPM & 0,18 & $<0,05$ \\
\hline ĐDLMDD với ĐDLMNT & 0,42 & $<0,05$ \\
\hline ĐDLMNT với ĐDLMTPM
\end{tabular}

Nhận xét: Bảng 3.3 cho thấy ĐDLMDD với ĐDLMTPM và ĐDLMDD với ĐDLMNT tương quan ở mức thấp; ĐDLMNT với ĐDLMTPM $(r=0,42 ; \mathrm{p}<0,05)$ tương quan ở mức trung bình, với $p<0,05$.

Bảng 3.4. Mối tương quan giữa sự tích tụ mỡ trong cơ thể với các chi số nhân trắc học ở bệnh nhân gan nhiếm mõ̃.

\begin{tabular}{|c|c|c|c|c|c|c|}
\hline \multirow{2}{*}{$\begin{array}{l}\text { Mõ̃ bụng } \\
\text { Chỉ tiêu/Chí số }\end{array}$} & \multicolumn{2}{|c|}{ ĐDLMDD } & \multicolumn{2}{|c|}{ ĐDLMTPM } & \multicolumn{2}{|c|}{ ĐDLMNT } \\
\hline & $\mathbf{r}$ & $\mathbf{p}$ & $\mathbf{r}$ & $\mathbf{p}$ & $\mathbf{r}$ & $\mathbf{p}$ \\
\hline Chiều cao & 0,08 & $>0,05$ & 0,11 & $>0,05$ & 0,24 & $<0,05$ \\
\hline Cân nặng & 0,01 & $>0,05$ & 0,02 & $>0,05$ & 0,48 & $<0,05$ \\
\hline Vòng bụng & 0,06 & $>0,05$ & 0,07 & $>0,05$ & 0,47 & $<0,05$ \\
\hline BMI & 0,05 & $>0,05$ & 0,07 & $>0,05$ & 0,42 & $<0,05$ \\
\hline
\end{tabular}

Nhận xét: Bảng 3.4 thấy có mối tương quan thuận ở mức trung bình giữa ĐDLMNT với các chỉ tiêu cân nặng $(r=0,48)$, vòng bụng $(r=0,47)$ và chỉ số $B M I(r=0,42)$ với $p<0,05$; tương quan thuận ở mức thấp giữa ĐDLMDD, ĐDLMTPM với chiều cao, cân nặng, vòng bụng và chỉ số BMI, với $p>0,05$.

\section{BÀN LUÂ̂N}

Có rất nhiều phương pháp đánh giá sự phân bố mõ như đo trọng lượng, chiều cao, VB, VM, tỷ VB/VM, BMI [4]. Trong nhiêu năm, tỉ lệ VB/VM (WHR) được sử dụng để đánh giá sự phân bố mõ cơ thể. Nhưng trong một số nghiên cứu, người ta đã thông báo rằng chu vi VB có liên quan mật thiết với sự phân bố mõ trung bình so với tỷ $\mathrm{VB} / \mathrm{VM}$.Tuy nhiên, phương pháp tốt nhất để đánh giá sự phân bố mõ là chụp cắt lớp vi tính. Phương pháp này không phải là phương pháp thường quy do phơi nhiễm phóng xạ và chi phí cao [5].

Gan nhiễm mõ thường phổ biến ở bệnh béo phì và liên quan đến mức độ béo phì. Sự phân phối mõ bất thường ở ổ bụng (trong bệnh béo phì nội tạng), là sự tăng tỉ lệ giữa vòng bụng và vòng mông, liên quan nhiều nhất đến mức độ thoái hoá mõ. Do đó, điều quan trọng là phải đánh giá mối liên quan giữa mức độ béo phì và mức độ nghiêm trọng của máu nhiễm mõ̃. Các nghiên cứu trước đây đã báo cáo một mối tương quan đáng kể giữa tình trạng nhiễm mõ gan và tỷ lệ eo/hông, điều này thể hiện tầm quan trọng của yếu tố dự báo nhiễm mỡ của gan (Kral et al., 1993) [theo 6].

Nhiều nghiên cứu đã đề câp đến $C T$ và MRI trong nghiên cứu sự phân bố chất béo trong bụng và tương quan lượng chất béo với các phép đo nhân trắc học, nhưng siêu âm vẫn là

một phương pháp dễ dàng và quan trọng để nghiên cứu sự phân bố chất béo.

Trong nghiên cứu của chúng tôi, cân nặng, vòng bụng, vòng mông và chỉ số $B M I$, tỳ lệ VB/VM đều tăng ở cả hai giới nhóm gan nhiễm mõ so với nhóm chứng.

Kết quả nghiên cứu này cũng phù hợp với nghiên cứu của tác giả Van Steenbergen và Lanckmans, tác giả Nuran Sabir và cs [6],[7].

Nghiên cứu của chúng tôi cho thây ĐDLMDD với ĐDLMTPM $(r=0,23 ; p<0,05)$ và ĐDLMDD với ĐDLMNT $(r=0,18 ; p<0,05)$ tương quan ở mức thấp; ĐDLMNT với ĐDLMTPM $(r=0,42 ; p$ $<0,05)$ tương quan ở mức trung bình. Điều này cho thấy mõ̃ nội tạng cho dữ liệu nhân trắc học chắc hơn mõ dưới da và mõ trước phúc mac.

Tương quan thuận ở mức trung bình giữa ĐDLMNT với các chỉ tiêu cân nặng $(r=0,48)$, vòng bụng $(r=0,47)$ và chỉ số BMI $(r=0,42)$ với $p<0,05$; tương quan thuận ở mức thấp giữa ĐDLMDD, ĐDLMTPM với chiều cao, cân nặng vòng bụng và chỉ số $B M I$, với $p>0,05$. Độ nhiễm mõ gan dày hơn tương quan đáng kể với cả dũ liệu nhân trắc học: cân nặng, chiều cao, vòng bụng và BIM, đặc biệt là độ dày lớp mõ̃ nội tạng. Kết quả nghiên cứu này cũng phù hợp với nghiên cứu của tác giả Van Steenbergen và Lanckmans, tác giả Nuran Sabir và cs [6],[7].

\section{KẾT LUÂN}

- Cân nặng, vòng bụng, vòng mông và chỉ số 
BMI, tỷ lệ VB/VM đều tăng ở cả hai giới nhóm gan nhiễm mõ̃ so với nhóm chứng.

- ĐDLMDD với ĐDLMTPM $(r=0,23 ; p<0,05)$ và ĐDLMDD với ĐDLMNT $(r=0,18 ; \mathrm{p}<0,05)$ tương quan ở mức thấp; ĐDLMNT với ĐDLMTPM $(r=0,42 ; p<0,05)$ tương quan ở mức trung bình.

- Tương quan thuận ở mức trung bình giữa ĐDLMNT với các chỉ tiêu cân nặng $(r=0,48)$, vòng bụng $(r=0,47)$ và chỉ số $B M I(r=0,42)$ với $p<0,05$; tương quan thuận ở mức thấp giữa ĐDLMDD, ĐDLMTPM với chiêu cao, cân nặng vòng bụng và chỉ số $B M I$, với $p>0,05$.

\section{TÀI LIÊUU THAM KHẢO}

1. Lê Văn Bàng (2004), "Tình hình béo phì ở đối tượng trên 15 tuổi tại thành phố Huế - Viêtt Nam", Kỷ yểu toàn văn các đề tài nghiên cứu khoa học Hội nghị Nội tiết \& Đái tháo đường toàn quốc lần thứ II, tr.666-674.

2. Suzuki R, Watanabe S, Hirai Y, et al (1993).
Abdominal wall fat index, estimated by ultrasonography, for assessment of the ratio of visceral fat to subcutaneous fat in the abdomen. Am J Med, 309-314.

3. Bô̂ Y tế: Các giá trị sinh học người Việt Nam bình thường thâap kỷ 90- Thế kỷ XX.. Nhà xuất bản $Y$ học Hà Nội, 2003.

4. Karacas P. P., Bozlir M. G. (2012), "Anthropometric indices in relation to overweight and obesity among Turkish medical students", Arch Med Sci, 8 (2), pp. 209-213

5. Silha J. V., Krsek M., Skrha J. V., et al (2003), "Plasma resistin, adiponectin and leptin level in lean and obese subjects: correlations with in insulin resistance", European Journal of Endocrinology, 149, pp. 331-335.

6. Nuran Sabir, Yurdaer Sermez, Selcuk Kazil, Mehmet Zencir (2001), "Correlation of abdominal fat accumulation and liver steatosis: importance of ultrasonographic and anthropometric measurements", European Journal of Ultrasound, pp 121-128.

7. Van Steenbergen W, Lanckmans S (1995), Liver disturbances in obesity and diabetes mellitus. Int J Obes, pp 27-36.

\title{
ĐÁNH GIÁ HIÊUU QUẢ KỸ THUÂT TIÊM NGOÀI MÀNG CỨNG DƯớI HƯớNG DẪN CẮT LỚP VI TÍNH Ở BÊNH NHÂN ĐAU DO THOÁT VỊ ĐĨA ĐỆM CộT SỐNG THẮT LƯNG
}

\author{
Phùng Anh Tuấn*, Nguyễn Xuân Khái*, \\ Dương Công Tuấn*, Nguyễn Đức Thuận*, Nguyễn Việt Dũng* \\ mức độ đau, mức độ mất chức năng sinh hoạt.

\section{SUMMARY} \\ THE EFFECTIVENESS OF CT-GUIDED \\ EPIDURAL STEROID INJECTIONS FOR LOW \\ BACK PAIN DUE TO LUMBAR DISC \\ HERNIATION
}

TÓM TẮT

Mục đích: Đánh giá hiệu quả của kỹ thuật tiêm ngoài màng cứng (NMC)dưới hướng dẫn cắt lớp vi tính (CVLT) trong điêu trị đau thắt lưng do thoát vị đĩa đệm (TVĐĐ). Đối tượng và phương pháp: 36bênh nhân (BN)đau thắt lưng do TVĐĐ được tiến hành tiêm NMC dưới hướng dẫn CVLT tại khoa $X$ quang can thiêp - Bệnh viện Quân y 103, từ 10/2019 đến tháng 09/2020. Đánh giá hiệu quả kỹ thuâtt bằng thang điểm đau VAS (visual analog scales) và thang điểm mất chức năng sinh hoạt ODI (Oswestry disability index). So sánh trước và sau tiêm bằng kiểm định $\mathrm{t}$ - test và Chi bình phương test. Kết quả: Điểm VAS giảm từ $6,11 \pm 0,89$ trước tiêm xuống còn $2,47 \pm 1,13$ sau tiêm 1 tháng. Điểm ODI giảm từ 64,67 \pm 10 trước tiêm xuống còn 24,89 $\pm 15,43$ sau tiêm 1 tháng. Khác biệt có ý nghĩa thống kê. Không cótai biến, biến chứng trong và sau khi thực hiện kỹ thuật. Kết luận: Tiêm NMC dưới hướng dẫn CLVTT là kỹ thuật an toàn và hiệu quả trong điều trị đau thắt lưng do TVĐĐ.

Tư khóa: Đau thắt lưng, tiêm ngoài màng cứng,

*Bệnh viện Quân y 103.

Chịu trách nhiệm: Nguyễn Việt Dũng,

Email: dung.nguyenviet.cdha@gmail.com

Ngày nhận bài: $1 / 2 / 2021$

Ngày phản biện khoa học: 23/2/2021

Ngày duyệt bài: 25/3/2021
Objects: Evaluating the effectiveness of epidural injection with corticoid under computed tomography guidance for treatment of low back paindue to disc herniation. Subjects and methods: 36 patients with low back pain due to disc herniation were CT - guided epidural injectedat the Interventional radiology department, 103 Military Hospitalfrom October 2019 to September 2020. All patients were evaluated by using VAS and ODI scores. Comparison of VAS and DOI scores pre -and 1 month post - injection were proceeded by $\mathrm{t}$ - test and chi square - test. Results: VAS score decreased from $6.11 \pm 0.89$ points before injection to $2.47 \pm 1.13$ points 1 month post - injection. ODI decreased from $64.67 \pm 10$ points before injection to $24.89 \pm 15.43$ points 1 month post - injection. The differences were significant. There were no complications. Conclusion: CT - guided epidural spinal injectionis a safe and effective procedure for treatmentof low back pain due to disc herniation.

Keywords: Low back pain, epidural spinal injection, visual analog scale, Oswestry disability index.

\section{I. ĐẶT VẤN ĐỀ}

